\title{
Automatic fracture optimization for shale gas reservoirs based on gradient descent method and reservoir simulation
}

\author{
Jiaheng Chen ${ }^{1,2}$, Lei Wang ${ }^{3 \oplus *}$, Cong Wang ${ }^{2}$, Bowen $\mathrm{Yao}^{2}$, Ye Tian ${ }^{2,4}$, Yu-Shu Wu ${ }^{2}$ \\ ${ }^{1}$ Research Institute of Petroleum Exploration \& Development, Beijing 100089, P. R. China \\ ${ }^{2}$ Department of Petroleum Engineering, Colorado School of Mines, Golden, CO 80401, USA \\ ${ }^{3}$ College of Energy, State Key Laboratory of Oil and Gas Reservoir Geology and Exploitation, Chengdu University of Technology, Chengdu \\ 610059, P. R. China \\ ${ }^{4}$ School of Petroleum Engineering, Southwest Petroleum University, Chengdu 610500, P. R. China
}

\section{Keywords:}

Gradient descent method

gas reservoir simulation

fracture spacing

fracture half-length

fracture conductivity

Cited as:

Chen, J., Wang, L., Wang, C., Yao, B., Tian, Y., Wu, Y.-S. Automatic fracture optimization for shale gas reservoirs based on gradient descent method and reservoir simulation. Advances in Geo-Energy Research, 2021, 5(2): 191-201, doi: 10.46690/ager.2021.02.08

\begin{abstract}
:
In shale gas reservoir development, determination of hydraulic fracture geometry for horizontal wells is a demanding yet challenging task. One type of approach for hydraulic fracture optimization is based on reservoir simulation. To improve optimization efficiency and accuracy, an automatic and robust procedure integrating the gradient descent method with gas reservoir simulation has been developed. Fractured reservoir models were constructed using the "Multiple INteracting Continua" method, whereby an in-house shale gas reservoir simulator was implemented to model multiple gas transport mechanisms including non-Darcy flow, gas desorption, Klinkenberg effect, and geomechanical effect. The optimization procedure was first validated against two ideal cases and then applied to two realistic cases to optimize fracture spacing, half-length, and dimensionless fracture conductivity. It showed that the optimization results depend on optimization objective, reservoir property, natural fractures, economics and termination criteria. This gradient descent assisted fracture optimization procedure can achieve significant computational reduction and high prediction accuracy for various shale gas reservoir cases.
\end{abstract}

\section{Introduction}

Hydraulic fracturing is a crucial technique for producing natural gas from shale and tight reservoirs. Energy Information Administration (EIA) estimated that natural gas production from hydraulically fractured wells makes up about two-thirds of total gas production in the U.S. (Perrin and Cook, 2016). Hydraulic fracturing is always a major capital expense on drilling and completion in unconventional gas reservoirs, for example, $32.9 \%$ of the $\$ 7.6 \mathrm{MM}$ needed for drilling and completing a single Marcellus Shale gas well is spent on hydraulic fracturing (Hefley and Seydor, 2011). Under such circumstances, a lot of attentions have been attracted to optimize fracture design, especially the three foremost parameters, fracture half-length, dimensionless fracture conductivity, and number of fracture stages (fracture spacing), which have a significant impact on gas recovery. However, optimal fracture design through simulation-based approach is a challenging task due to real geological uncertainty. In most scenarios, the delivered project design is far from the optimal one since not all of the parameter combinations could be exhausted with current numerical tools (Ma et al., 2015).

Quite a few optimization techniques have been introduced to solve problems in the oil and gas industry, such as recovery processes, facility planning, history matching, well placement and operation, and so on. These approaches can be classified into two groups: gradient-based and gradientfree approaches. Gradient-free approaches adopt the values of objective function instead of calculating the gradient of objective function (gradient-based) to search for the optimal solution, which is a heuristic technique. For example, Bangerth et al. (2006) compared gradient-based methods (Simultaneous Perturbation Stochastic Approximation and Finite Difference

\section{Yandy
Scientific}

Press
${ }^{*}$ Corresponding author.

E-mail address: jiaheng.chen@petrochina.com.cn (J. Chen); wanglei@cdut.edu.cn (L. Wang); tornadoco@163.com (C. Wang);

byao@mines.edu (B. Yao); tian220y@gmail.com (Y. Tian); ywu@mines.edu (Y. Wu). 2207-9963 (c) The Author(s) 2021.

Received April 6, 2021; revised April 20, 2021; accepted April 20, 2021; available online April 24, 2021. 
Gradient) and gradient-free method (Very Fast Simulated Annealing (VFSA)) in solving well placement problems. Asadollahi and Naevdal (2009) used steepest descent and conjugate direction methods (gradient-based) to optimize water flooding problem. Hajizadeh et al. (2009) introduced an ant colony optimization algorithm for automatic history matching. Chen et al. (2012) also investigated history matching problem by applying three derivative-free algorithms (VFSA, Simultaneous Perturbation and Multivariate Interpolation, and Quadratic Interpolation Model-based Algorithms). Bellout et al. (2012) optimized well placement using derivative-free methods based on pattern search. Cui (2016) utilized the improved particle swarm optimization (PSO) technique for solving the post-stack impedance inversion problem.

Hydraulic fracturing optimization has been analyzed by several studies. However, most experimental and simulation studies are basically sensitivity analyses. That is, one specific parameter is examined while keeping other parameters constant to seek the relationship between the specific parameter and the objective, few studies investigated multiple parameters simultaneously. For instance, Brake (2013) concluded that longer fractures perform better in Wamsutter field in southwest Wyoming after analyzing historical fracturing data, performance and surveillance data, and field trials, but did not provide the optimal length. Saldungaray and Palisch (2012) investigated hydraulic fracture optimization with Barnett Shale case studies by focusing on fracture geometry and conductivity, and reached that optimal fracture spacing increases with increasing conductivity.

A few studies investigated fracture optimization based on mathematical optimization and reservoir simulation using real reservoir properties. Yu and Sepehrnoori (2013) used quadratic response surface methodology to optimize a pair of hydraulically fractured horizontal wells in Barnett Shale. Shale gas production was modeled using well distance, three hydraulic fracture variables, porosity and permeability to obtain the maximized Net Present Values (NPV) with different natural gas prices. Gas desorption is considered using Langmuir isotherm that is replicated by solution gas ratio in a black oil model. Rammay and Awotunde (2016) integrated the differential evolution algorithm with a commercial reservoir simulator to maximize the NPV for shale gas reservoirs in terms of hydraulic fracture length, spacing, and conductivity. Langmuir gas desorption, stress dependent conductivity and local grid refinement were incorporated into a dual-porosity flow model. Zhang and Sheng (2020) constructed a fracture optimization framework that used a modified PSO algorithm to seek for the optimal NPV. Stimulated reservoir volume calculated based on both displacement discontinuity method and natural fracture failure criterion was proposed and realized as "Multiple INnteracting Continua" (MINC) models to optimize fracture half-length and spacing on Eclipse 300. It's found that NPV is a better objective for fracture optimization than stimulated reservoir volume, which doesn't indicate the best economic scenario. Several aspects can be conducted to further improve fracture optimization. For example, to increase accuracy of results by employing optimization techniques that can flexibly expand the diversity of fit equations, and to achieve more realistic results by gas reservoir simulations that can take into account Klinkenberg effect and effect of geomechanics. Therefore, an automatic fracture optimization procedure integrating the gradient descent method (GDM) with an in-house simulator that can account for multiple gas transport effects was put forward.

GDM, a popular optimization technique, is selected to solve the fracturing optimization problem for several reasons. First, GDM is a mathematically proven method that uses realtime first order derivative in the computation process. That is, the next search direction is determined upon instantaneous gradients, which can greatly reduce the number of simulations. Second, there is no need to fit equations between variables and objective functions. Third, there are quite a few optional algorithms, such as conjugate gradient method, making it flexible to trial other algorithms. The weakness of GDM is that a global solution cannot be guaranteed if the problem and its constraints are nonconvex. In this case, algorithms can be restarted with systematically selected initial points to obtain better solutions. Provided these advantages, GDM is chosen for fracture optimization.

In this paper, the fracture optimization problem for horizontal wells with geological uncertainty is first formulated. Then we present the GDM, its variants, search criteria, and the established optimization procedure. This new optimization procedure is further validated with existing models. Finally, two case studies based on real field data are illustrated with concluding remarks.

\section{Problem formulation}

For a typical optimization problem with a real-valued function $f\left(x_{0}, x_{1}, x_{2}, \cdots, x_{n}\right)$, optimization seeks to minimize or maximize $f$ subject to $x_{0}, x_{1}, x_{2}, \cdots, x_{n} \in \Omega$, where $\Omega$ is a subset of the domain. $f$ is called objective or loss function, and $\Omega$ are often termed as feasible solutions, among which that minimizes or maximizes the objective function is called an optimal solution. The problem can be unconstrained or constrained depending on $\Omega$. If $\Omega$ is defined by a system of equations or inequalities, then the problem is constrained, and vice versa. In petroleum engineering, common constraints are facility capacity, operation safety and economics (Asadollahi and Naevdal, 2009). Theoretically, fracture optimization is a nonconvex and unconstrained problem.

For fracture optimization, gradient calculation with respect to fracture geometry is not straightforward. Thus indirect adjoint equation of profit related to fracture geometry variables is devised to implement gradient-based algorithms. There are three common economic models (cash flow, financial, and tax), which define costs in different ways and result in different profit values. The cash flow model is selected seeing that it includes the time value of money (Thompson and Wright, 2015), which is defined as:

$$
P=\frac{F}{(1+r)^{n}}
$$

where $P$ is present value, $F$ is future value, $r$ is the currency interest rate for a specific period, and $n$ is the number of per- 


$\begin{aligned} & \text { Gross Production } \\ & - \text { Shrinkage } \\ = & \text { Gross Sales } \\ & \times \text { Net Revenue Interest } \\ = & \text { Net Sales } \\ & \times \text { Price } \\ = & \text { Gross Revenue } \\ & - \text { Severance \& Ad Valorem Taxes } \\ & - \text { Windfall Profits Tax } \\ & - \text { Operating Costs } \\ & - \text { Loan Interest Payments } \\ = & \text { Net Operating Income BFIT (Before Federal Income Tax) } \\ & - \text { Investments } \\ & - \text { Loan Principal Payments } \\ = & \text { Net Operating Income AFIT (After Federal Income Tax) }\end{aligned}$

Fig. 1. Natural gas asset cash flow diagram (Thompson and Wright, 2015).

iods. The detailed cash flow diagram for an oil and gas asset is shown in Fig. 1. NPV is computed by summing up all cash flows and discounting to a specific time with a discount rate. Finally, the objective value, referred to as cumulative discounted NPV (CDNPV), can be obtained by adding these NPVs to an interested time.

After the terms of cost function and its calculation steps are set up, independent variables need to be determined. Stimulated reservoir volume is typically optimized by choosing proper horizontal lateral length, number of fracture stages, fracture isolation technique, and job size (Saldungaray and Palisch, 2012). Herein, fracture half-length, dimensionless conductivity, and number of fracture stages (as a measure of fracturing spacing) are selected as independent variables for fracture optimization.

The general discounted NPV function is formulated as:

$$
\begin{aligned}
& f_{T}\left(n_{\text {stage }} \text { or } H F_{s p}, H F_{h l}, F_{c d}\right)= \\
& -\int_{0}^{T}\left\{\sum_{j=i}^{N_{p}}\left[w_{1} p_{g} q_{g}(t)-w_{2} p_{w, d i s p} q_{w}(t)\right]\right. \\
& \left.-\sum_{k=1}^{N}\left[w_{3} C_{h f}\left(n_{\text {stage }}, H F_{h l}, F_{c d}\right)+w_{4} C_{\text {other }}(t)\right]\right\}(1+r)^{-n} d t
\end{aligned}
$$

where $q_{g}$ and $q_{w}$ are production rates of gas and water, respectively; gas price $p_{g}=\$ 2.436 / \mathrm{Mscf} ; p_{w, \text { disp }}$ is the cost for disposing water; $j$ represents different production sources; $C_{\text {other }}(t)$ is the drilling cost if time $t$ is year one and is additional investment after year one; $N$ represents multiple wells; $w_{1}, w_{2}, w_{3}$ and $w_{4}$ are weighting factors to scale their corresponding items' influence; $n_{\text {stage }}$ is number of hydraulic fracture stages and $H F_{s p}$ is hydraulic fracture spacing; $H F_{h l}$ is hydraulic fracture half-length; $F_{c d}$ is dimensionless fracture conductivity. Since cost of hydraulic fracturing operations varies, it is separated out from other expenses as an individual term, denoted as $C_{h f}$. $C_{h f}$ is a function of optimization parameters, occurring only at year one (Ma et al., 2015):

$$
C_{h f}=\frac{C_{t \max }}{n_{\max }} n_{\text {real }}+a_{1}\left(1+\frac{a_{2} F_{c d}+1000}{1500}\right)\left(2 H F_{h l}\right)^{a_{3}}
$$

where $C_{t \max }$ is the cost at the maximum number of stages $n_{\max } ; n_{\text {real }}$ is the real stage numbers during the optimization process, which is equal to reservoir length divided by hydraulic fracture spacing; $a_{1}, a_{2}$ and $a_{3}$ are coefficients controlling the sensitivity of $C_{h f}$ to $F_{c d}$ and $H F_{h l}$.

\section{GDM}

Gradient descent is a first order iterative optimization algorithm for finding the minimum of an unconstrained problem. It descends by taking steps in the opposite direction of the function gradient at a given point. The Taylor series of a continuously differentiable function is:

$$
\begin{gathered}
f\left(x_{k}+\alpha_{k} d_{k}\right)=f\left(x_{k}\right)+\alpha_{k} g_{k}^{T} d_{k}+o\left(\alpha_{k}\right), \quad \alpha_{k}>0 \\
g_{k}^{T} d_{k}=\left\|g_{k}\right\|\left\|d_{k}\right\| \cos \bar{\theta}_{k}
\end{gathered}
$$

where $x_{k}$ is an independent optimization parameter at $k^{\text {th }}$ point, $d_{k}$ is the search direction at $k^{\text {th }}$ point, step size $a_{k}$ is the amount of advance of $x_{k}$ in each iteration, $g_{k}^{T}$ is the gradient of $f$ at $x_{k}$, higher-order terms of $\alpha_{k}$ is $o\left(\alpha_{k}\right)$ which can be ignored, $\bar{\theta}_{k}$ is the angle between $g_{k}$ and $d_{k}$. Thus, if we want to obtain the minimum value of $f, g_{k}^{T} d_{k}$ should be minimized. That is, $\cos \bar{\theta}_{k}=-1, \bar{\theta}_{k}=\pi$, so $d_{k}$ should be opposite to $g_{k}^{T}$.

Since CDNPV was calculated from a gas reservoir simulator implicitly, numerical rather than analytical differentiation was adopted to calculate the derivatives. Here, central difference scheme was used (Young and Mohlenkamp, 2009):

$$
\frac{\partial f}{\partial x_{i}}=\frac{f\left(x_{i}+h\right)-f\left(x_{i}-h\right)}{2 h}
$$

Armijo inexact line search is used to solve the step size. For $\beta \in(0,1), \sigma \in(0,0.5)$, assume $\alpha_{k}=\beta^{m_{k}}$, where $m_{k}$ is the minimum integer that satisfies the following inequality:

$$
f\left(x_{k}+\beta^{m_{k}} g_{k}^{T} d_{k}\right) \leq f x_{k}+\sigma \beta^{m_{k}} g_{k}^{T} d_{k}
$$

It can be proved that under the condition that $f(x)$ is differentiable and $g_{k}^{T} d_{k}$ is negative, there is always a positive $\sigma$ such that a sufficiently large positive integer $m_{k}$ ensures the inequality. Ensuring the inequality by choosing the proper step size is the guarantee of searching in the right direction for the optimal objective from previous iteration. By following the Armijo rule, we can find the step size and then update the next search. Specific steps of Armijo rule are listed below:

Step 0: initialize $\beta \in(0,1), \sigma \in(0,0.5)$, and $m=0$;

Step 1: if the inequality above (Eq. (7)) is true, then assign $m_{k}=m, x_{k+1}=x_{k}+\beta^{m_{k}} d_{k}$, otherwise go to step 2;

Step 2: $m=m+1$, go to step 1 .

Besides gradient and step size, Euclidean norm of a vector, as one of the algorithm termination criteria, is calculated:

$$
\|x\|_{2}=\sqrt{\sum_{i=1}^{N_{v}} x_{i}^{2}}
$$




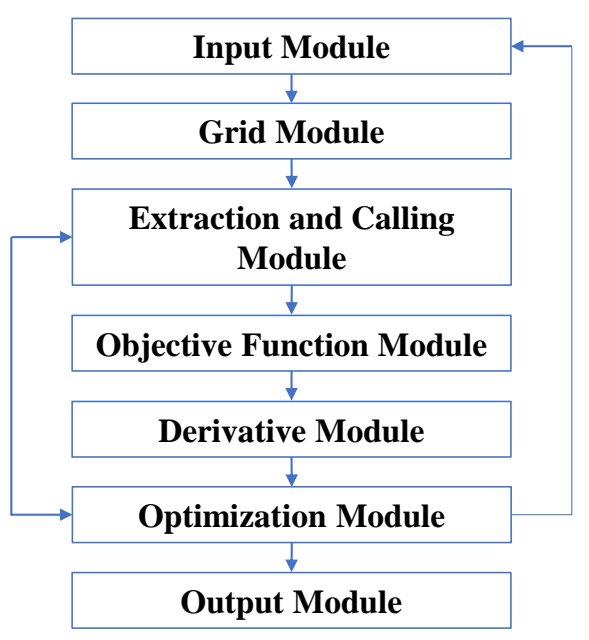

Fig. 2. Relationships of modules in the optimization procedure.

GDM is powerful for solving convex problems; however, it has limitations for nonconvex problem since it relies on the initial point. Different initial points might lead to different local optimal values (Goodfellow et al., 2016). Therefore, it is preferred to run the algorithm with multiple initial points to obtain better results and reduce errors.

\section{Optimization procedure}

In order to combine a reservoir simulator with the optimization algorithm, a variety of modules have been established, including input, output, optimization, objective function, grid, derivative, and extraction and calling modules. The relationships among these modules are displayed in Fig. 2. Here, only the Multiphase Subsurface FLOW (MSFLOW) reservoir simulator, MINC method of the objective function module, and meshing technique of the grid module are introduced.

\subsection{MSFLOW simulator}

MSFLOW is a reservoir simulator developed for modeling flow of oil, gas and water in fractured reservoirs ( $\mathrm{Wu}, 2015)$. It is adapted to model gas and water flow for fracture optimization in unconventional reservoirs. Isothermal condition is used for shale gas reservoir simulation seeing that inclusion of heat exchange does not show much difference (Moridis et al., 2010). The modified MSFLOW incorporates the following characteristics that have been recognized for controlling flow in unconventional gas reservoirs (Wang et al., 2017):

- Nonlinear and multiphase flow (Darcy and non-Darcy flow);

- Modified permeability due to Klinkenberg effect;

- Nonlinear gas adsorption/desorption;

- Effect of geomechanics on fracture and matrix permeability.

\subsection{MINC method}

MSFLOW simulator employs a generalized multicontinuum approach to model interactions and flows between

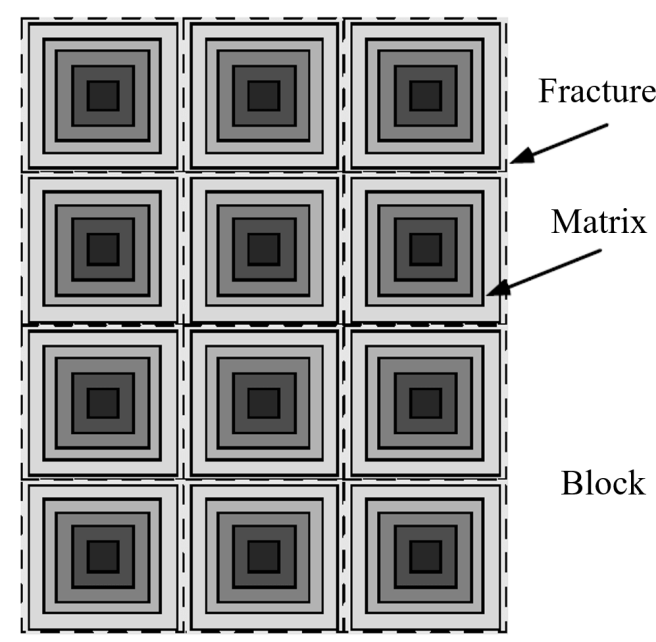

Fig. 3. Relationships of modules in the optimization procedure.

fracture and matrix, including the double-porosity method (Warren and Root, 1963), the multiple porosity method (Wu and Pruess, 1998), the dual-permeability method (Pereira et al., 2006), and the MINC method (Pruess, 1983). MINC partitions a "primary" reservoir grid into "secondary" nested grids. It is much more rigorous than classical double-porosity approach, which mainly assumes pseudo-steady state flow. The reason is that it describes pressure gradients between matrix and fracture by appropriately sub-partitioning matrix blocks. MINC approach assumes that fluid pressure invades the matrix block slowly but propagates through the fractures rapidly, meaning that the condition of matrix is determined by local distance from fracture. Thus, matrix and fracture can be treated as one-dimensional strings of nested grid blocks (Pruess, 1991), as shown in Fig. 3.

MINC method can accommodate "dual-permeability" model with certain accuracy sacrifice since both matrix and fracture are approximated as one grid block and thus pseudosteady state flow should be assumed. However, the model should work fine under steady-state due to the minimal gradients near matrix surfaces. MINC is applicable for reservoirs, in which fracture systems are not so sparse. Specifically, for two interacting continua, MINC method is the same as doubleporosity and dual-permeability models.

\subsection{Automatic meshing technique}

Local grid refinement was applied to structured grids intersected by fractures at the scale of fracture width. The fractures become a set of small size and high permeability grids, and thus numerical accuracy can be improved.

A logarithmic refinement meshing code was created and implemented in the original Cartesian meshing module of MSFLOW to achieve automatic meshing of multiple hydraulic fractures at desired locations with specific widths, lengths and heights. The logarithmic refinement can be done in the X, Y and $\mathrm{Z}$ directions and the size of the mesh is controlled by a parameter $a_{m}$. To accelerate reservoir simulation and fracture optimization, refinement is only conducted in $\mathrm{X}$ direction and at the tips of hydraulic fractures in $\mathrm{Y}$ direction. Also, hydraulic 


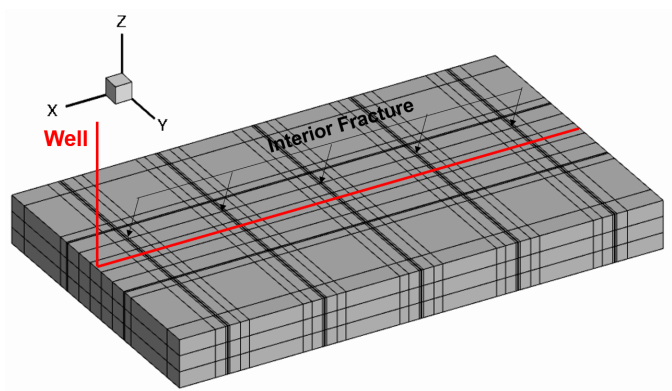

(a)

Fig. 4. Original model and simplified reservoir model for validation.

Table 1. Parameters of the simplified reservoir model.

\begin{tabular}{lll}
\hline Parameter & Value & Unit \\
\hline Matrix porosity & 0.06 & \\
Hydraulic fracture porosity & 0.5 & \\
Matrix permeability & $9.87 \times 10^{-19}$ & $\mathrm{~m}^{2}$ \\
Dimensionless fracture conductivity & 20 & \\
Hydraulic fracture permeability & $3.948 \times 10^{-14}$ & $\mathrm{~m}^{2}$ \\
Hydraulic fracture half-length & 100 & $\mathrm{~m}$ \\
Reservoir length & 1000 & $\mathrm{~m}$ \\
Reservoir width & 600 & $\mathrm{~m}$ \\
Reservoir thickness & 100 & $\mathrm{~m}$ \\
Initial reservoir pressure & $4.14 \times 10^{7}$ & $\mathrm{~Pa}$ \\
Constant bottom hole pressure & $1.0 \times 10^{6}$ & $\mathrm{~Pa}$ \\
Initial gas saturation & 1 & \\
Gas density (standard) & 0.668 & $\mathrm{~kg} / \mathrm{m}^{3}$ \\
Gas viscosity (standard) & $5.955 \times 10^{-6}$ & $\mathrm{~Pa} \cdot \mathrm{s}$ \\
Klinkenberg coefficient & $1.03 \times 10^{6}$ & $\mathrm{~Pa}$ \\
Langmuir volume & $2.2 \times 10^{-3}$ & $\mathrm{~m}{ }^{3} / \mathrm{ton}$ \\
Langmuir pressure & $1.5759 \times 10^{7}$ & $\mathrm{~Pa}$ \\
Total compressibility of all media & $1.0 \times 10^{-9}$ & $1 / \mathrm{Pa}$ \\
Reservoir temperature & 50 & ${ }^{\circ} \mathrm{C}$ \\
\hline
\end{tabular}

fractures are uniformly spaced. Another feature of this meshing module is that it can identify matrix, hydraulic fracture, and well based on their location information.

\section{Model validation}

\subsection{Validation of the simplified reservoir model}

For validation, the simplified reservoir geometry containing one-half of a hydraulic fracture with one fracture spacing was used. Then the final production for the whole reservoir was computed using Eq. (9), assuming the hydraulic fractures are symmetrically distributed. To validate this simplified model, a case producing only gas is established, of which the main parameters are provided in Table 1.

$$
\operatorname{prod}_{\text {total }}=2 \operatorname{prod}_{1 / 2} \frac{\text { Reservoir Length }}{H F_{s p}}
$$

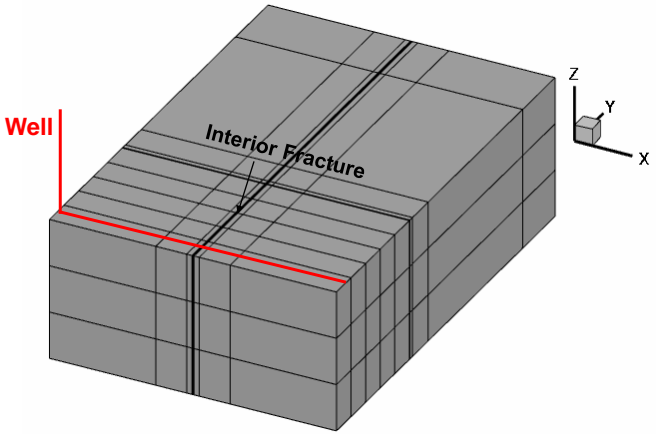

(b)

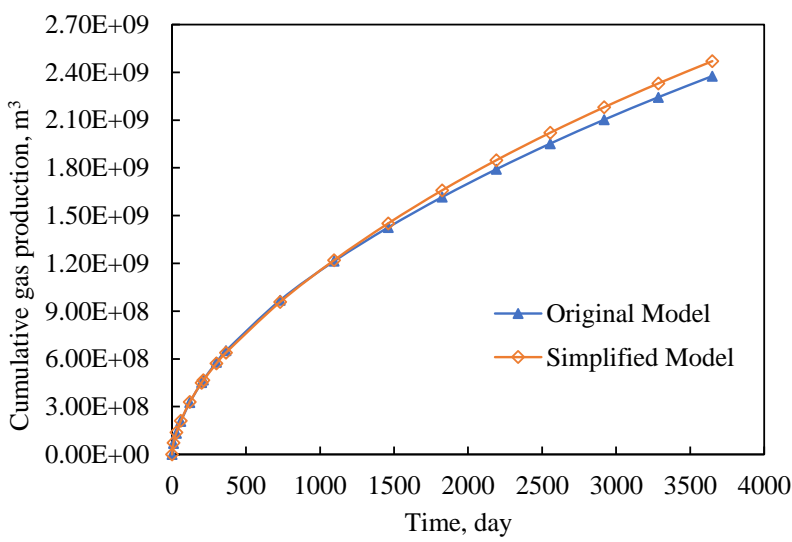

Fig. 5. Ten-year cumulative gas production for the original and simplified models.

Fig. 4 shows the original and simplified five-hydraulicfracture reservoir geometries. Simulation results using Intel Core $^{\mathrm{TM}}$ i5-4300M CPU of $2.60 \mathrm{GHz}$ with $8 \mathrm{~GB}$ memory indicated that it took 63.23 seconds to finish the simulation on the original model and only 8.64 seconds on the simplified model. As compared in Fig. 5, two reservoir geometries demonstrated satisfactory agreement with each other. The deviation of the simplified model from the original model in terms of predicted gas production at 10 years is $3.95 \%$, which is acceptable.

Besides, gas reservoir pressure distributions of the original and simplified models are compared in Figs. 6 and 7, proving that these two models undergo highly consistent pressure depletion at different times. Therefore, the simplified reservoir model can be used as a surrogate for optimization analysis. In addition, the contours indicate linear flow between matrix and hydraulic fracture and radial flow near the fracture tip, leading to elliptic pressure distribution.

\subsection{Validation of the optimization procedure}

The optimization procedure is verified by two cases. The first one seeks the minimum value for an explicit function with two variables. The second one sets the one-year cumulative gas production as the objective function for a fractured gas reservoir and maximizes gas production in terms of fracture stages, half-length, and dimensionless conductivity without co- 


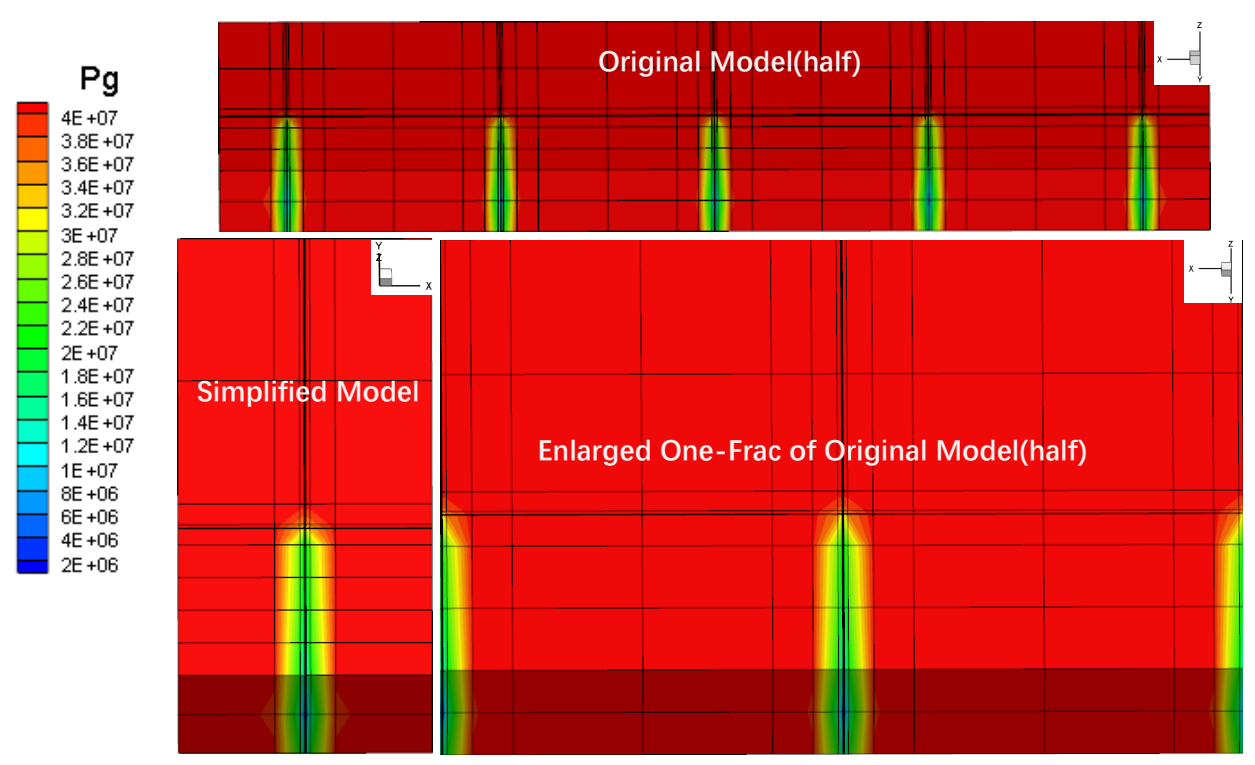

Fig. 6. Gas pressure distributions for original and simplified reservoir models at 10 days.

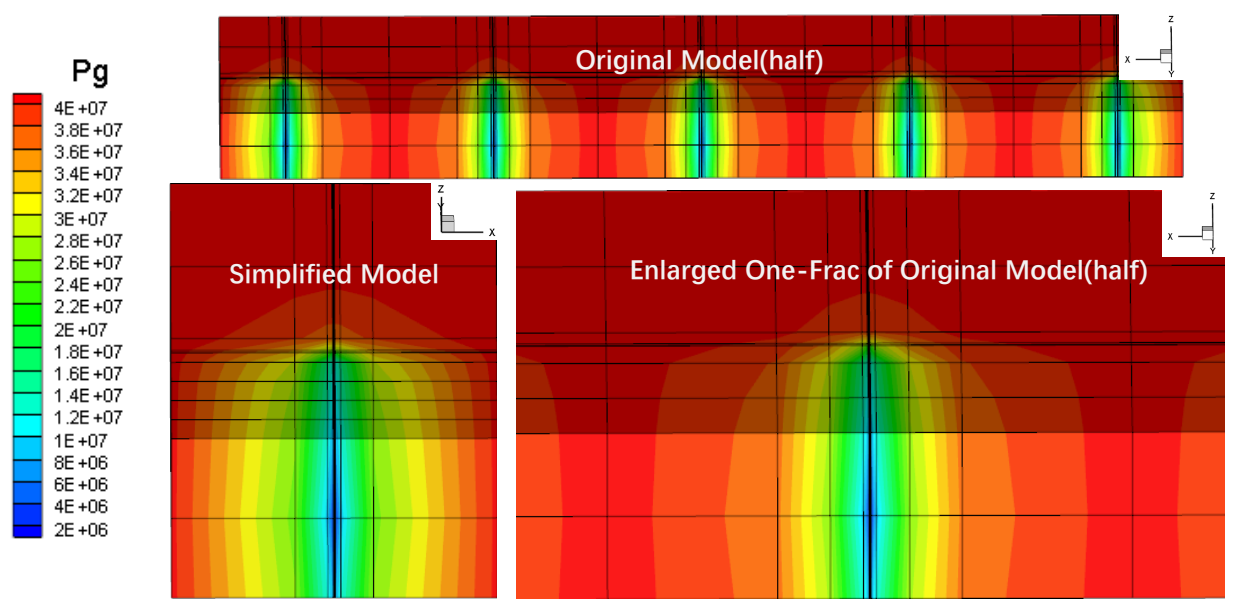

Fig. 7. Gas pressure distributions for original and simplified reservoir models at 120 days.

Table 2. Validation results of the optimization system with an explicit function.

\begin{tabular}{lll}
\hline Initial point & Number of iterations & Objective function value \\
\hline$(-1,1)$ & 18 & $6.35 \mathrm{E}-22$ \\
$(-10,10)$ & 20 & $2.48 \mathrm{E}-22$ \\
$(-20.80,30.42)$ & 21 & $6.71 \mathrm{E}-23$ \\
$(-150,600)$ & 22 & $2.18 \mathrm{E}-22$ \\
$(1000,3000)$ & 23 & $6.06 \mathrm{E}-22$ \\
\hline
\end{tabular}

st constraints.

\subsubsection{Validation case \#1}

Function $f=3 x^{2}+4 y^{2}$ is an elliptic paraboloid with a minimum value of 0 at $(0,0)$ in $3 \mathrm{D}$ space. It was coded into the gradient descent optimizat ion procedure and different initial points were tested. As shown in Table 2, no matter what the initial point is, the algorithm always finds the minimum value with a few iterations.

\subsubsection{Validation case \#2}

To verify the optimization procedure for a real reservoir (Table 1), reservoir simulator MSFLOW was integrated to transform fracture spacing, half-length, and dimensionless conductivity into the objective-gas production. The lower boundaries of hydraulic fracture spacing, half-length, and dimensionless fracture conductivity are $20 \mathrm{~m}, 0 \mathrm{~m}$, and 0 $\mathrm{m}$, respectively, represented by [20 00 . The corresponding upper boundaries are [500 300 100]. Then the test began with a random initial point [400 150 5]. To visualize the evolving process of three optimization parameters, they are plotted against the calling times of MSFLOW simulator (Fig. 8), gas production (Fig. 9), and program running time (Fig. 10). 


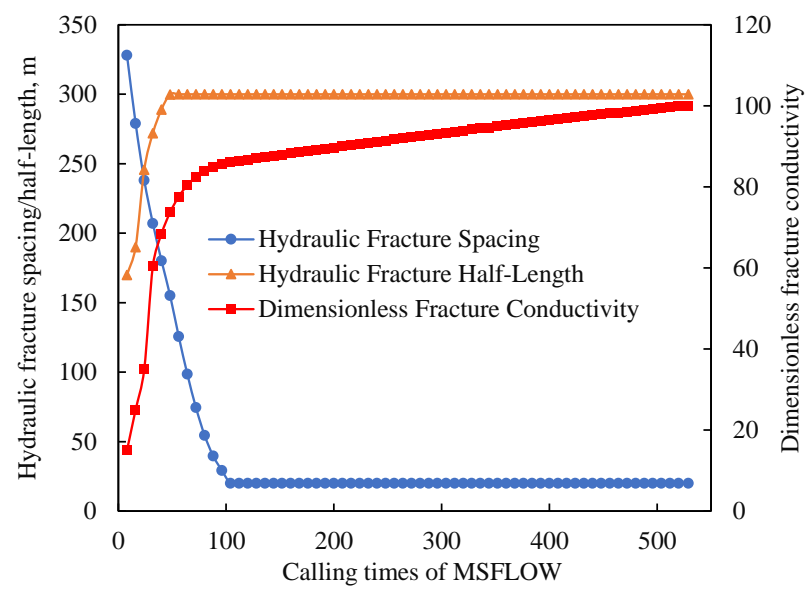

Fig. 8. Hydraulic fracture spacing, half-length and dimensionless conductivity vs. calling times of MSFLOW with initial point [400 150 5].

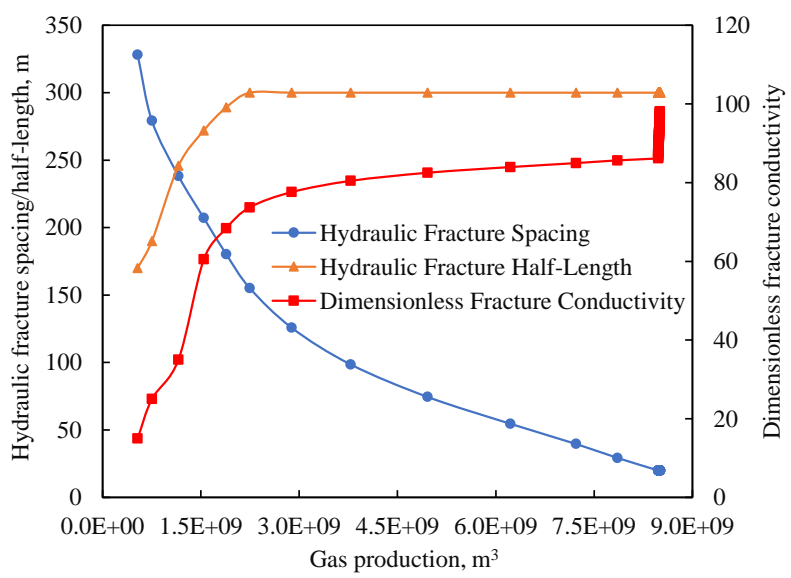

Fig. 9. Hydraulic fracture spacing, half-length and dimensionless conductivity vs. gas production when optimization starts from initial point [ [400 150 5].

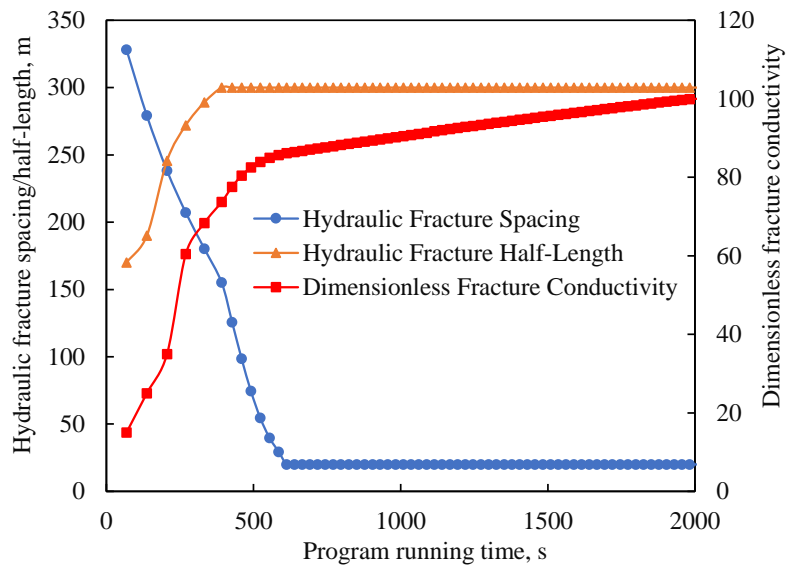

Fig. 10. Hydraulic fracture spacing, half-length and dimensionless conductivity vs. optimization procedure running time with initial point [400 150 5].

Fig. 9 shows that the maximum gas production of $8.5079 \times 10^{9} \mathrm{~m}^{3}$ is achieved when hydraulic fracture spacing, half-length, and dimensionless fracture conductivity are 20 $\mathrm{m}, 300 \mathrm{~m}$, and $100 \mathrm{~m}$, respectively. In other words, when hydraulic fracture spacing is minimized (i.e., stage number is maximized) as well as fracture half-length and dimensionless conductivity reach their maxima, gas production can be maximized. This verifies the monotonic know-how that without cost constraints, it is better to have more stages, longer fracture half-length, and higher dimensionless conductivity.

In Fig. 10, both the optimal hydraulic fracture spacing and half-length were quickly found by the GDM, but the optimal dimensionless conductivity took longer, due to the varying sensitivity regarding gas production. A common observation is that these parameters change rapidly in the early stage of optimization before leveling out.

Multiple random initial points were assigned to validate the optimization procedure, turning out that all of them found the same optimal values of gas production. In addition, we calculated gas production using some random parameter combinations, all of which yield values lower than the optimal gas production found at [20 300 100].

\section{Results and discussion}

The optimization procedure has been applied to two real reservoir cases, including: 1) a basic unconventional gas reservoir case (same as Validation case \#2); and 2) an unconventional gas reservoir with natural fractures. The CDNPV is used as objective function and the simulation time is extended to ten years.

Optimization parameters are bounded by real reservoir conditions. The number of fracture stages ranges from 2 to 50 based on statistics from Marcellus and Delaware gas fields (U.S. EIA, 2016). The number of fracture stages is replaced by fracture spacing, seeing that fracture stages can only be integers while objective function is required to be differentiable. Fracture spacing ranges from 20 to $500 \mathrm{~m}$. Fracture half-length varies from 0 to $300 \mathrm{~m}$ and dimensionless fracture conductivity is set within $[0,100]$.

\subsection{Case study \#1}

Case study \#1 is the same as Validation case \#2, except that CDNPV is the objective. Drilling cost is set as \$2 MM based on Marcellus gas well data (U.S. EIA, 2016), and $C_{t \max }$ is set as \$2 MM too. $a_{1}, a_{2}$, and $a_{3}$ are generally chosen based on field application, engineers' experience and overall investment control. $a_{1}, a_{2}$, and $a_{3}$ are set as 10, 0.04 and 1.8, thus cost of hydraulic fracturing becomes ( $\$ 0.080$ to $\$ 3.672 \mathrm{MM}$ ):

$$
C_{h f}=40,000 n_{\text {real }}+10\left(1+\frac{0.04 F_{c d}+1000}{1500}\right)\left(2 H F_{h l}\right)^{1.8}
$$

When the norm of the gradient is less than 0.05 or the changes of three optimization parameters between two steps are less than 5E-5, the optimization process will be terminated. These termination criteria influence the computation time for optimization and the degree of accuracy.

A series of initial points are designed so that a large domain could be covered, and the results for six combinations are li- 
Table 3. Optimal results starting from various initial points for Case \#1.

\begin{tabular}{lllllll}
\hline Initial points & $H F_{s p}(\mathrm{~m})$ & $H F_{h l}(\mathrm{~m})$ & $F_{c d}$ & CDNPV $(\$)$ & Calling times of MSFLOW & Program running time $(\mathrm{s})$ \\
\hline$\left[\begin{array}{llll}300 & 150 & 5\end{array}\right]$ & 27.00 & 293.26 & 100 & 4919317 & 1336 & 7994.2 \\
{$\left[\begin{array}{llll}480 & 290 & 90\end{array}\right]$} & 27.20 & 293.95 & 100 & 4915165 & 452 & 3552.8 \\
{$\left[\begin{array}{llll}400 & 20 & 70\end{array}\right]$} & 27.27 & 293.68 & 100 & 4920558 & 620 & 5118.7 \\
{$\left[\begin{array}{llll}100 & 50 & 60\end{array}\right]$} & 27.34 & 292.67 & 100 & 4916353 & 806 & 4853.9 \\
{$\left[\begin{array}{llll}250 & 200 & 40\end{array}\right]$} & 27.23 & 293.90 & 100 & 4915283 & 528 & 3239.8 \\
{$\left[\begin{array}{llll}25 & 200 & 90\end{array}\right]$} & 27.20 & 293.83 & 100 & 4915511 & 666 & 3767.9 \\
\hline
\end{tabular}

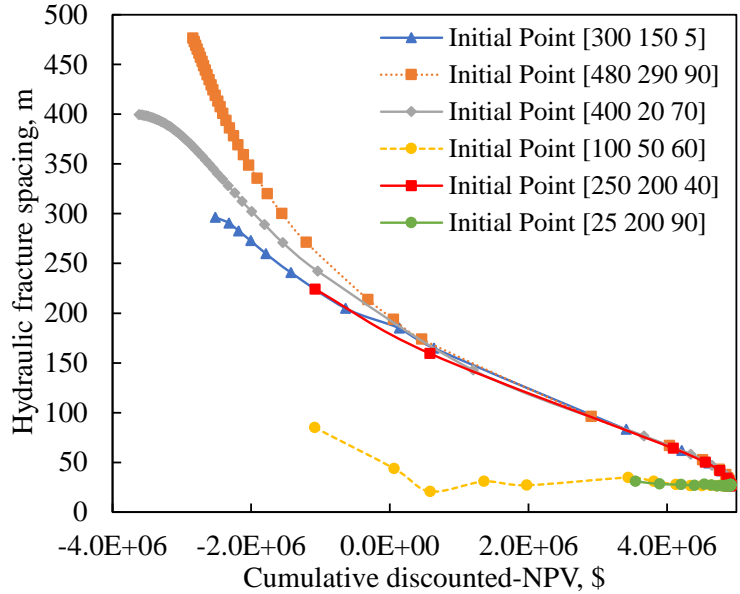

Fig. 11. Optimization process of fracture spacing vs. CDNPV with different initial points.

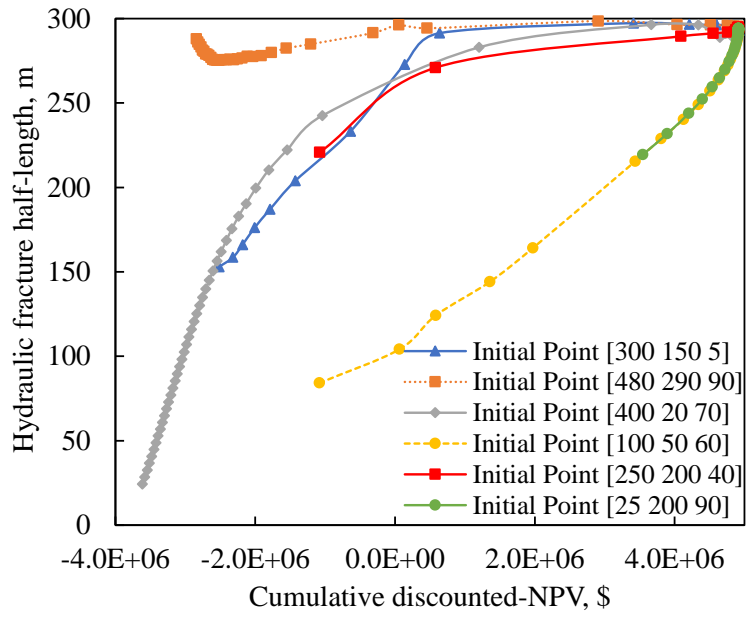

Fig. 12. Optimization process of fracture half-length vs. CDNPV with different initial points.

sted in Table 3. It is clear that no matter what the initial point is, the maximum CDNPV and the corresponding hydraulic fracture spacing, half-length, and dimensionless conductivity converge well. The best economic scenario with the gas price of $\$ 2.436 / \mathrm{Mscf}$ is found from initial point [400 20 70], which is $\$ 4.92 \mathrm{MM}$ with $H F_{s p}, H F_{h l}$, and $F_{c d}$ values of $27.27 \mathrm{~m}$, $293.68 \mathrm{~m}$, and $100 \mathrm{~m}$.

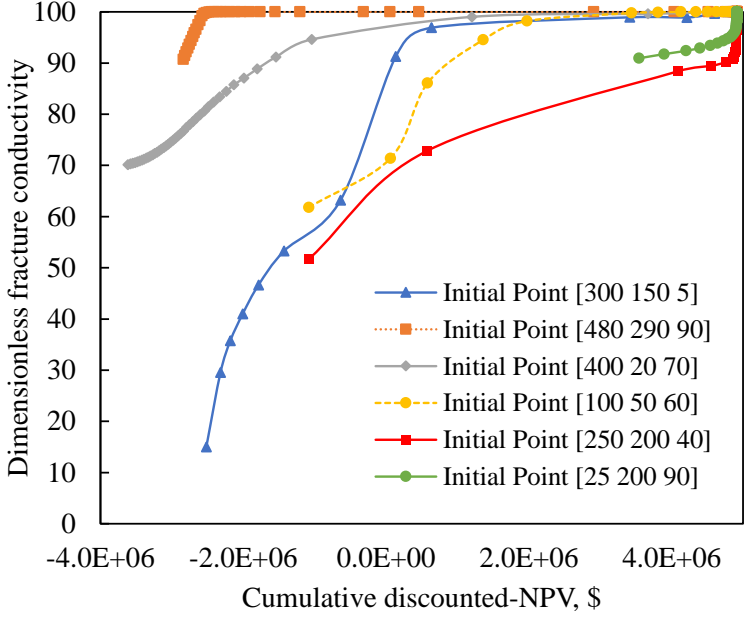

Fig. 13. Optimization process of dimensionless fracture conductivity vs. CDNPV with different initial points.

The values of hydraulic fracture spacing, half-length, and dimensionless conductivity during the optimization processes with various initial points are plotted with CDNPV in Fig. 11 to Fig. 13, respectively. All tests indicate that the maximum CDNPV converges around \$4.92 MM. The corresponding values of three optimization parameters are overall reasonable, reflecting the economical constraint on hydraulic fracture spacing and fracture half-length. In Fig. 13, $F_{c d}$ hits the boundary, because its weighting factor $a_{2}$ was set too small such that increasing $F_{c d}$ increases gas production or CDNPV by a larger amount than $C_{h f}$.

\subsection{Case study \#2}

To better understand the influence of natural fractures on optimization, case study \#2 is designed. Global flow is assumed to occur only through the hydraulic fracture continuum, while matrix and all fractures interact locally by means of "inter-porosity" flow (double-porosity model). The mesh generation is different from previous cases because of natural fractures. The MINC method partitions reservoir grids into nested meshes, the intermediate layers of which are then assigned with natural fracture properties. The MINC parameters and natural fracture properties are listed in Table 4.

Table 5 presents the results for seven initial points, from which the maximum CDNPV is found to be $\$ 6.28 \mathrm{MM}$ with 
Table 4. MINC model parameters for the reservoir with natural fractures.

\begin{tabular}{lll}
\hline Parameter & Value & Unit \\
\hline Natural fracture permeability & $k_{N F x}=k_{N F y}=k_{N F z}=9.87 \times 10^{-16}$ & $\mathrm{~m}^{2}$ \\
Natural fracture porosity & $\phi_{N F}=0.4$ & \\
MINC type & Three sets of parallel infinite fractures & \\
MINC volume fraction & $1 \mathrm{E}-6$ & \\
\hline
\end{tabular}

Table 5. Optimal results starting from various initial points for Case \#2.

\begin{tabular}{lllllll}
\hline Initial points & $H F_{s p}(\mathrm{~m})$ & $H F_{h l}(\mathrm{~m})$ & $F_{c d}$ & CDNPV $(\$)$ & Calling times of MSFLOW & Program running time (s) \\
\hline$\left[\begin{array}{llll}250 & 50 & 50\end{array}\right]$ & 102.92 & 73.32 & 49.26 & 6280993 & 1092 & 16556 \\
{$\left[\begin{array}{llll}100 & 200 & 35\end{array}\right]$} & 101.27 & 74.71 & 38.68 & 6281190 & 1150 & 15457 \\
{$\left[\begin{array}{llll}400 & 120 & 22\end{array}\right]$} & 100.77 & 75.55 & 41.04 & 6281017 & 1130 & 15778 \\
{$\left[\begin{array}{llll}500 & 180 & 90\end{array}\right]$} & 102.24 & 73.32 & 88.69 & 6280462 & 1010 & 15760 \\
{$\left[\begin{array}{llll}22 & 280 & 5\end{array}\right]$} & 101.29 & 76.72 & 0.923 & 6280976 & 1282 & 18535 \\
{$\left[\begin{array}{llll}310 & 250 & 1\end{array}\right]$} & 103.63 & 76.01 & 0.49 & 6280953 & 772 & 9675 \\
{$\left[\begin{array}{llll}50 & 20 & 72\end{array}\right]$} & 100.44 & 73.97 & 71.91 & 6280938 & 252 & 3231 \\
\hline
\end{tabular}

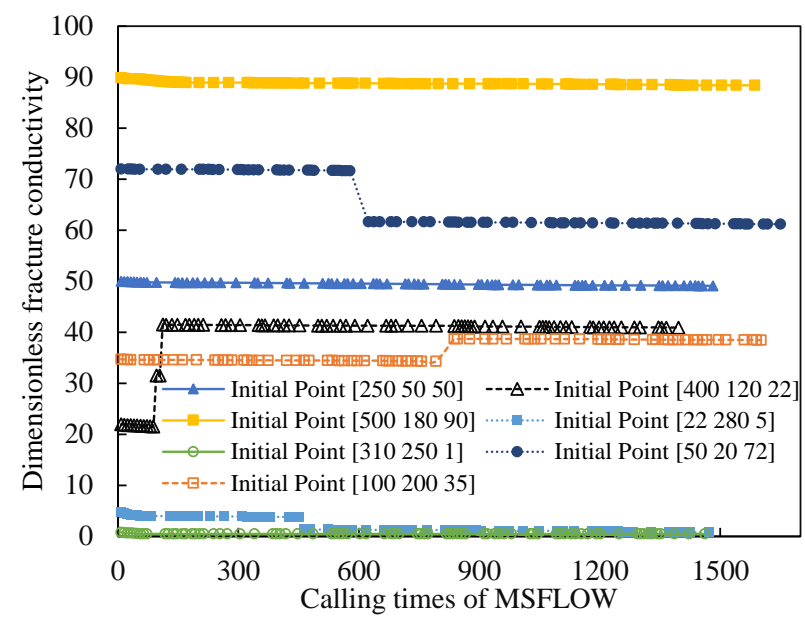

Fig. 14. Dimensionless fracture conductivity vs. calling times of MSFLOW for the natural fracture case with various initial points.

$H F_{s p}=101.27 \mathrm{~m}$ and $H F_{h l}=74.71 \mathrm{~m}$. Compared with $\mathrm{CDNPV}=\$ 4.92 \mathrm{MM}, H F_{s p}=27.27 \mathrm{~m}$, and $H F_{h l}=293.68$ $\mathrm{m}$ in Case study \#1, natural fractures significantly improve CDNPV by $27.64 \%$ as well as result in much bigger fracture spacing and shorter half-length. However, for dimensionless fracture conductivity, whichever initial point is used, it is always trapped locally to a trivial solution, as shown in Fig. 14. The reason is that due to the existence of natural fractures, hydraulic fracture shows no effect on production once it exceeds a threshold conductivity, which needs to be further investigated.

Random fracture conductivity values were tested with the same hydraulic fracture spacing and half-length values to determine the range of the threshold value. Fig. 15 displays three example tests with similar trends. CDNPV first increases

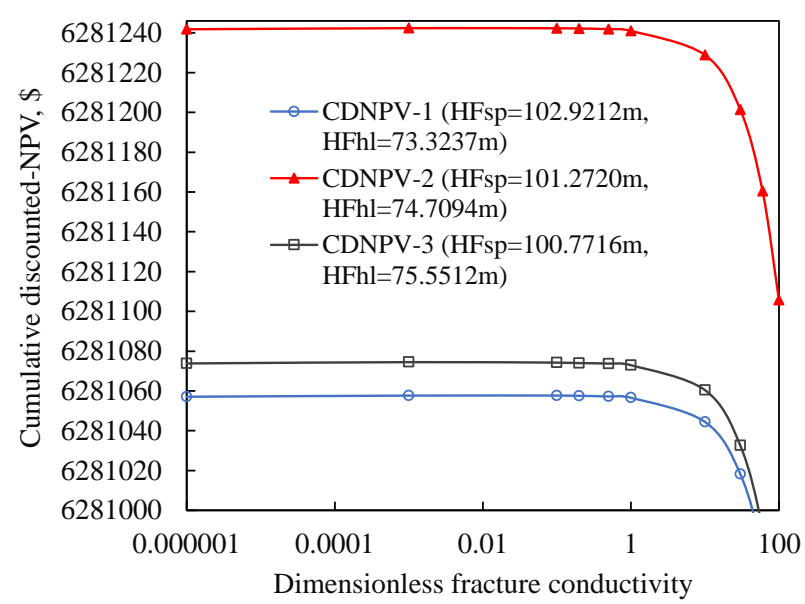

Fig. 15. Effect of dimensionless fracture conductivity on CDNPV for fixed hydraulic fracture spacing $H F_{s p}$ and half-length $H F_{h l}$.

very slightly (barely noticeable) when $F_{c d}$ is smaller than their threshold values, and then decreases. For all tested cases, threshold values are below 0.2 , which is minute in comparison to the natural fracture permeability. Eventually, the maximum CDNPV obtained is $\$ 6.2812 \mathrm{MM}$ with $H F_{s p}=101.27 \mathrm{~m}$, $H F_{h l}=74.71 \mathrm{~m}$ and $F_{c d}=0.001 \mathrm{~m}$.

It should be mentioned that when three optimization parameters approach their optimal values with large step sizes, fluctuations occur owing to the overshooting characteristics of GDM. In Case study \#2, fluctuations of hydraulic fracture spacing and half-length along with the times of calling MSFLOW are observed for different initial points, as shown in Fig. 16. Overall, starting from different initial points, hydraulic fracture spacing and half-length can converge steadily to the optimal values with acceptable fluctuations. 


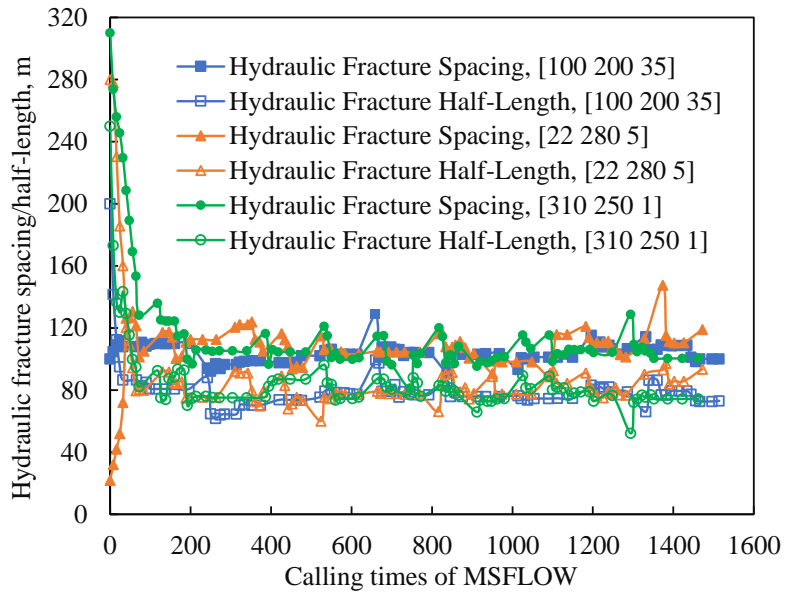

Fig. 16. Fluctuations of hydraulic fracture spacing and half-length during optimization.

\section{Conclusions}

An automatic hydraulic fracture optimization procedure is developed and validated for unconventional gas reservoir development. Based on case studies, we can draw the following conclusions:

- In case cost is not a constraint, gas production is a monotonically increasing function of hydraulic fracture spacing, half-length, and conductivity. Taking economics into account, hydraulic fracture spacing and half-length converge to realistic values.

- Natural fractures contribute notably to economic development of unconventional gas reservoirs, resulting in dramatic change of the optimal hydraulic fracture spacing, half-length, and conductivity.

- This optimization procedure that integrates gradient decent method with a gas reservoir simulator demonstrates satisfactory numerical stability and high accuracy.

The gradient descent algorithm could be easily substituted by other gradient-based methods for comparison and future study. This optimization procedure is useful for optimizing hydraulic fracturing design for unconventional gas reservoirs.

\section{Nomenclature}

$a_{i}=$ Sensitivity coefficient, dimensionless

$C_{\text {other }}(t)=$ Drilling cost if time $t$ is year 1 , additional investment after year one, $\$$

$C_{h f}=$ Hydraulic fracturing costs, $\$$

$C_{t \max }=$ The cost at the maximum number of stages, $\$$

$d_{k}=$ Search direction at $k^{\text {th }}$ point, dimensionless

$f=$ Function

$F=$ Future value, $\$$

$F_{c d}=$ Dimensionless fracture conductivity, dimensionless

$g_{k}^{T}=$ Gradient of objective function at $x_{k}$, dimensionless

$H F_{h l}=$ Hydraulic fracture half-length, $\mathrm{m}$

$H F_{s p}=$ Hydraulic fracture spacing, $\mathrm{m}$

$k=$ Permeability, $\mathrm{m}^{2}$

$m_{k}=$ The iteration number when a smaller function value

is found, dimensionless

$n=$ Number of periods, dimensionless $n_{\max }=$ Maximum number of fracture stages, dimensionless

$n_{\text {real }}=$ Real number of fracture stages, dimensionless

$o(\alpha)=$ Higher-order infinitesimal of $\alpha$, dimensionless

$P=$ Present value, $\$$

$p_{g}=$ Gas price, $\$ / \mathrm{Mscf}$

$p_{w, \text { disp }}=$ Cost for disposing water, $\$ / \mathrm{m}^{3}$

$r=$ Currency interest rate, dimensionless

$w_{i}=$ Weighting factor, dimensionless

$x_{k}=$ Independent variable at $k^{\text {th }}$ point, dimensionless

$\|x\|_{2}=$ Euclidean norm, dimensionless

$\alpha_{k}=$ Step size of independent variables, dimensionless

$\alpha_{m}=$ Controlling parameter for mesh size refinement, dimensionless

$\beta=$ Parameter for Armijo rule, dimensionless

$\bar{\theta}_{k}=$ The angle between $g_{k}$ and $d_{k}$, degree

$\phi=$ Porosity, dimensionless

$\sigma=$ Parameter for Armijo rule, dimensionless

AFIT $=$ After federal income tax

BFIT = Before federal income tax

CDNPV = Cumulative discounted net present value, $\$$

EIA = Energy Information Administration

GDM $=$ Gradient descent method

MINC = Multiple interacting continua

MSFLOW = Multiphase subsurface flow model

NPV $=$ Net Present Value, $\$$

PSO = Particle swarm optimization

VFSA = Very Fast Simulated Annealing

\section{Acknowledgement}

The corresponding author thanks the supports from the National Natural Science Foundation of China (Nos. 52074040, U20A20265) and the Sichuan Science and Technology Program (No. 2021YJ0355).

\section{Conflict of interest}

The authors declare no competing interest.

Open Access This article is distributed under the terms and conditions of the Creative Commons Attribution (CC BY-NC-ND) license, which permits unrestricted use, distribution, and reproduction in any medium, provided the original work is properly cited.

\section{References}

Asadollahi, M., Naevdal, G. Waterflooding optimization using gradient based methods. Paper SPE 125331 Presented at SPE/EAGE Reservoir Characterization \& Simulation Conference, Abu Dhabi, UAE, 19-21 October, 2009.

Bangerth, W., Klie, H., Wheeler, M., et al. On optimization algorithm for the reservoir oil well placement problem. Computational Geosciences, 2006, 10: 303-319.

Bellout, M. C., Echeverría Ciaurri, D., Durlofsky, L. J., et al. Joint optimization of oil well placement and controls. Computational Geosciences, 2012, 16: 1061.

Brake, A. C. Fracture optimization in a giant gas field. Paper SPE 164029 Presented at SPE Unconventional Gas Conference and Exhibition, Muscat, Oman, 28-30 January, 2013.

Chen, C., Jin, L., Gao, G., et al. Assisted history matching using three derivative-free optimization algorithms. Paper 
SPE 154112 Presented at Spe Europec/eage Conference, Copenhagen, Denmark, 4-7 June, 2012.

Cui, X. Poststack impedance inversion using improved particle swarm optimization. Paper Presented at Society of Exploration Geophysicists SEG Technical Program Expanded Abstracts 2016, Dallas, Texas, 16 October-21 October, 2016.

Goodfellow, I., Bengio, Y., Courville, A. Deep Learning: Adaptive Computation and Machine Learning Series, 2016 Edition. Massachusetts, USA, The MIT Press, 2016.

Hajizadeh, Y., Christie, M. A., Demyanov, V. Ant colony optimization algorithm for history matching. Paper SPE 121193 Presented at Society of Petroleum Engineers EUROPEC/EAGE Conference and Exhibition, Amsterdam, Netherlands, 8-11 June, 2009.

Hefley, B., Seydor, S. The economic impact of the value chain of a Marcellus shale well. Social Science Electronic Publishing, 2011.

Ma, X., Gildin, E., Plaksina, T. Efficient optimization framework for integrated placement of horizontal wells and hydraulic fracture stages in unconventional gas reservoirs. Journal of Unconventional Oil and Gas Resources, 2015, 9: 1-17.

Moridis, G. J., Blasingame, T. A., Freeman, C. M. Analysis of mechanisms of flow in fractured tight-gas and shalegas reservoirs. Paper SPE 139250 Presented at SPE Latin American and Caribbean Petroleum Engineering Conference, Lima, Peru, 1-3 December, 2010.

Pereira, C. A., Kazemi, H., Ozkan, E. Combined effect of non-Darcy flow and formation damage on gas well performance of dual-porosity and dual-permeability reservoirs. SPE Reservoir Evaluation \& Engineering, 2006, 9(5): 543-552.

Perrin, J., Cook, T. Hydraulically fractured wells provide twothirds of U.S. natural gas production. 2016. (accessed 6 April, 2021)

Pruess, K. GMINC-A Mesh Generator for Flow Simulations in Fractured Reservoirs. Berkeley, USA, Lawrence Berkeley National Laboratory, 1983.

Pruess, K. TOUGH2-A General-Purpose Numerical Simulator for Multiphase Fluid and Heat Flow. Berkeley, USA,
Lawrence Berkeley Laboratory, 1991.

Rammay, M. H., Awotunde, A. A. Stochastic optimization of hydraulic fracture and horizontal well parameters in shale gas reservoirs. Journal of Natural Gas Science and Engineering, 2016, 36: 71-78.

Saldungaray, P. M., Palisch, T. T. Hydraulic fracture optimization in unconventional reservoirs. Paper SPE 96812 Presented at SPE Middle East Unconventional Gas Conference and Exhibition, Abu Dhabi, UAE, 23-25 January, 2012.

Thompson, R., Wright, J. Oil and Gas Property Evaluation, 2015 Edition. Colorado, USA, Thompson-Wright Associates, 2015.

U.S. Energy Information Administration. March 2016. Trends in U.S. Oil and Natural Gas Upstream Costs.

Wang, L., Wang, S., Zhang, R., et al. Review of multi-scale and multi-physical simulation technologies for shale and tight gas reservoirs. Journal of Natural Gas Science and Engineering, 2017, 37: 560-578.

Warren, J. E., Root, P. J. The behavior of naturally fractured reservoirs. Society of Petroleum Engineers Journal, 1963, 228: 245-255.

Wu, Y. S. Multiphase Fluid Flow in Porous and Fractured Reservoirs, First Edition. Amsterdam, Netherlands, Gulf Professional Publishing, 2015.

Wu, Y. S., Pruess, K. A numerical method for simulating nonNewtonian fluid flow and displacement in porous media. Advances in Water Resources, 1998, 21: 351-362.

Young, T., Mohlenkamp, M. J. Introduction to numerical methods and Matlab Programming for Engineers. Lecture 27: 101-103, Ohio University Department of Mathematics, OH, 2009.

Yu, W., Sepehrnoori, K. Optimization of multiple hydraulically fractured horizontal wells in unconventional gas reservoirs. Journal of Petroleum Engineering, 2013, 2013: $1-16$.

Zhang, H., Sheng, J. Optimization of horizontal well fracturing in shale gas reservoir based on stimulated reservoir volume. Journal of Petroleum Science and Engineering, 2020, 190: 107059. 